Introduction

\title{
The Treatment of Distal Radius Fractures in the Elderly
}

Guillaume Herzberg, MD, $\mathrm{PhD}^{1}$

${ }^{1}$ Wrist Surgery Unit, Herriot Hospital, Lyon, France

J Wrist Surg 2015;4:149.

The optimal treatment of a severely displaced intra-articular distal radius fracture (DRF) in elderly patients remains controversial. Closed reduction and casting is the most traditional option. This simple treatment can provide a good functional outcome, but the healing period is long, and the residual bony malalignment may be significant. This may lead to a poor outcome especially in elderly patients who are independent in the activities of daily living.

Alternatives to closed treatment have been proposedincluding external fixation, percutaneous pinning, and locking volar plate fixation-but all of these techniques have drawbacks in this patient population.
In this Special Focus Section, the reader will find a new concept for treatment of DRF in the elderly patient. Rather than attempting to fix an irreparable articular fracture, an immediate wrist hemiarthroplasty is performed. This concept is based on the experience in the shoulder where irreparable fractures have been treated with a primary arthroplasty. This Special Focus issue explores this concept and provides some preliminary results, as well as technical pearls, about the use of wrist arthroplasty for posttraumatic arthritis associated with distal radius malunion.
DOI http://dx.doi.org/ $10.1055 / \mathrm{s}-0035-1558830$. ISSN 2163-3916. 